\title{
DIGITAL IMAGE COMPRESSION TECHNIQUES
}

\author{
Gomathi.K.V ${ }^{1}$, Lotus. $R^{2}$ \\ ${ }^{1}$ Centre for Information Technology and Engineering, Manonmaniam Sundaranar University, Tirunelveli, Tamil \\ Nadu, India \\ ${ }^{2}$ Centre for Information Technology and Engineering, Manonmaniam Sundaranar University, Tirunelveli, Tamil \\ Nadu, India
}

\begin{abstract}
In today's world of electronic revolution, digital images play an important role in consumer electronics. The need for storing images grows day to day. Compression of digital images plays an important role in storing the images. Due to compression the memory occupied by the images gets reduced and so that we can store more images in the available memory space. Also compression results in encoding of images which itself offer a security on transaction. This paper deals with a clear note on various aspects regarding compression and various compression techniques used and their implementation.
\end{abstract}

Keywords: digital image, compression, data redundancy, compression techniques.

\section{INTRODUCTION}

Image: An image is the result of a visual signal of any object captured by optical devices such as human eyes, camera lenses etc., Image is the two dimensional representation of the object.

Digital image: Image when stored in electronic devices such as computers, it is known as the digital image. Thus a digital image is the numeric representation of an image. Digital images are of two types - raster and vector. Raster images refers to the images built using a finite set of digital values, called picture elements or pixels. Pixel is the smallest element in an image. The value that is equivalent to the colour of that specific pixel is stored in the place of the pixel. Images stored in electronic devices fall under this category. Vector images refer to the images that are resulted from mathematical calculations. Vector refers to the elements that have both magnitude and direction (Raster images have only magnitude). In electronic devices, vector type is used to refer texts stored as images or to store images that require more accuracy even in zoomed level. Vectors have both magnitude and direction. So the images are clear even when zoomed. Usually the term digital image refers to raster images.

Need for Compression: The need for storage of digital information increases rapidly with the growth of internet technology. Images are widely used in applications like medical, army and satellite images. Everyday an enormous amount of information is stored, processed and transmitted digitally [4]. The unprocessed or raw digital images occupy more space. This necessitates compression of data. Image compression is the process of representing the required information with the possible minimal amount of data. Hence it is useful for reducing the amount of memory space need for the file. Thus for the growing requirements of image storage and digital technology, storing the compressed images allows us to store more images in the given disk space. Also transmission rate of images gets increased due to compression. This paper deals with the process of compression in the second part, Compression types in the Third part, Compression techniques in the Fourth part and gets concluded in the fifth part.

\section{COMPRESSION PROCESS}

\section{Compression}

Compression is achieved by removing one or more of the three basic data redundancies. They are

$>$ Coding Redundancy

$>$ Interpixel Redundancy

$>$ Psychovisual Redundancy.

Data redundancy refers to the data that are in the state of being not or no longer needed.

\subsection{Coding Redundancy}

A code is a set of symbols that represents some information in the image. As a method of compression, codes occurring many times are represented with lesser bits and other codes are represented with more number of bits. In that, coding redundancy is the form of data redundancy that is present when less than optimal code words are used to represent any information[1] and so this redundancy deals with the data representation of the image. Let us consider that we have a gray image which is represented as $R$ then the average number of bits or optimal value of codes required to represent each pixel is given by

$$
\mathrm{N}_{\mathrm{avg}}=\sum_{\mathrm{i}=0}^{\mathrm{N}-1} \mathrm{M}\left(\mathrm{r}_{\mathrm{i}}\right) \mathrm{p}_{\mathrm{r}}\left(\mathrm{r}_{\mathrm{i}}\right)
$$

Where $r_{i}$ represents the kth gray level in the image. $M\left(r_{i}\right)$ is the number of occurrence of $r_{k}, p_{r}\left(r_{i}\right)$ - probability of $\mathrm{r}_{\mathrm{k}}$ to occur and $\mathrm{N}$ is the total number of gray levels in the image. Coding redundancy is removed using Huffman coding. 


\subsection{Interpixel Redundancy:}

Interpixel redundancy is the form of data redundancy that is related to interpixel correlation within an image [11]. While storing an image, it is not necessary to store all the details of pixels in the image. This is allowed due to the reason that the value of any pixel can be calculated from the values of the neighboring pixels. This is known as interpixel dependency. Interpixel correlation is the difference between the information content and the information capacity of the image. In an image, consequent pixels may contain same colour or relative shades of same colfour. Instead storing the full details of all the pixels the values of correlated pixels may be calculated from the details of a single pixel for which full details are stored. Thus this data redundancy works on image representation.

Let us consider any two pixels which are represented as functions $\mathrm{f}(\mathrm{r})$ and $\mathrm{g}(\mathrm{r})$ respectively. The formula used to find the correlation between those two pixels is given by the convolution theorem,

$$
f(r) \circ g(r)=\int_{-\infty}^{\infty} f^{*}(a) g(r+a) d a \quad \rightarrow(B)
$$

Depending upon the value of the equation (B) we can say that the two pixels are correlated or not. A Threshold $\mathrm{T}$ is fixed and if the value of (B) is greater than $T$ then the pixels are not correlated else the pixels are correlated. If the pixels are auto-correlated then we have $f(r)=g(r)$. The value of correlated pixels can be calculated based on two operations namely repetition and prediction. Repetition is used for auto-correlated pixels and prediction is used for other correlated pixels. This means the relatively or equally valued pixels are calculated. This method can be applied for both colour and grayscale images. Interpixel redundancy is of two types - spatial and Interframe.

Spatial Redundancy:Spatial redundancy refers to redundancy in still images. Consider any pixel, A having coordinates $(\mathrm{x}, \mathrm{y})$, then Value $(\mathrm{x}, \mathrm{y})$ always depends on $\left(\mathrm{x}^{\prime}, \mathrm{y}^{\prime}\right)$ and the Value( $\left.x^{\prime}, y^{\prime}\right)$, for all $\left(x^{\prime}, y^{\prime}\right)$ belongs to the neighbourhood of $(x, y)$.

Interframe redundancy: Interframe redundancy refers to the redundancy present in videos. Consider the set of frames $\left(\mathrm{x}, \mathrm{y}, \mathrm{t}_{\mathrm{i}}\right)$, for all $\mathrm{i}=0,1,2, \ldots$ then Value $\left(\mathrm{x}, \mathrm{y}, \mathrm{t}_{\mathrm{i}}\right)$ is related to each other.

Interpixel redundancy is removed using compression techniques like Constant area coding, Run length encoding.

\subsection{Psychovisual Redundancy:}

Psychovisual redundancy is the data redundancy that works with respect to image content. The psychovisual redundancy refers to the information that is not noticed or processed by the human eyes. This is because certain information has more importance for Human Visual System(HVS) than others based upon the fact that Human eye has the effect of recognizing only low frequencies and neglecting high frequencies. Thus its function as a low pass filter is used by compression algorithms, to reduce the probably high frequency signal.

By removing the psychovisually redundant information, the compression achieved is lossy but the end user who is a human being, cannot find the difference.

To find the psychovisually redundant information, a discrimination threshold $(\Delta \mathrm{I})$ is found for all the pixels and this value is added to the initial value of the pixel $I_{i}$. After that, the pixel having smallest value is the psychovisually redundant information.

Discrimination threshold is calculated using the Weber's Law

$$
\Delta \mathrm{I}_{\mathrm{i}}=\mathrm{K}_{\mathrm{w}} \cdot \mathrm{I}_{\mathrm{i}}
$$

where $K_{w}$ is a constant known as the Weber's fraction. i.e the value of $\Delta \mathrm{I}$ always depends upon the $\mathrm{I}_{\mathrm{i}}$.

The equation (C) may be rewritten as $\Delta \mathrm{I}_{\mathrm{i}}=\mathrm{K}_{\mathrm{w}} \mathrm{I}_{\mathrm{i}}+0$ which is similar to the equation of a straight line $\mathrm{y}=\mathrm{mx}+$ $\mathrm{b}$ with $\mathrm{K}_{\mathrm{w}}$ as the slope and $\mathrm{b}$ value as 0 . Thus we can see all the values of $\Delta \mathrm{I}_{\mathrm{i}}$ lies in a straight line when plotted using a graph. Lossy compression algorithms like JPEG compression works to remove this type of redundancy.

\section{COMPRESSION TYPES}

Compression process always results in encoding of the input. Then to retrieve the original image decompression is used. Based on whether the original image can be retrieved from the compressed file, the types of compression are

1. Lossless compression

2. Lossy compression

\subsection{Lossless Compression Technique:}

In lossless compression technique, the original image is perfectly retrieved from the compressed image. This is also known as noiseless coding. The lossless compression techniques follow the entropy encoding schemes. In entropy encoding, the nature of the input data is analyzed and the encoding or further process is performed based on it.

\subsubsection{Basic Steps in Lossless Compression}

Technique:

Lossless compression is done in following steps:

1. Analysis and grouping of data.

2. Encoding. 


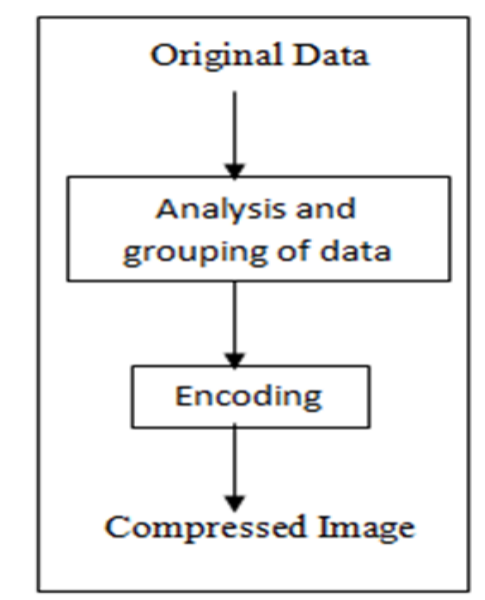

Fig-1: Lossless compression process

\section{Analysis and Grouping of data:}

The input data is analyzed and grouped together based on the compression technique involved.

\section{Encoding:}

The compression technique is applied in this step to the previously grouped or analyzed data.

\subsection{Lossy Compression Technique}

In lossy compression technique the original images are not exactly reconstructed after decompression. But the output produced will be reasonably close to the original image. Only data that is considered trivial is deleted. The remaining data will be sufficient to represent the required information. Also in lossy compression schemes there are more possibilities for higher compression ratios. The lossy compression techniques follow source encoding schemes. In source encoding, the nature of the input data is not needed to be analyzed.

\subsubsection{Basic Steps in Lossy Compression:}

Any lossy compression process is done in three steps:

1. Matrix Transformation

2. Quantization

3. Encoding

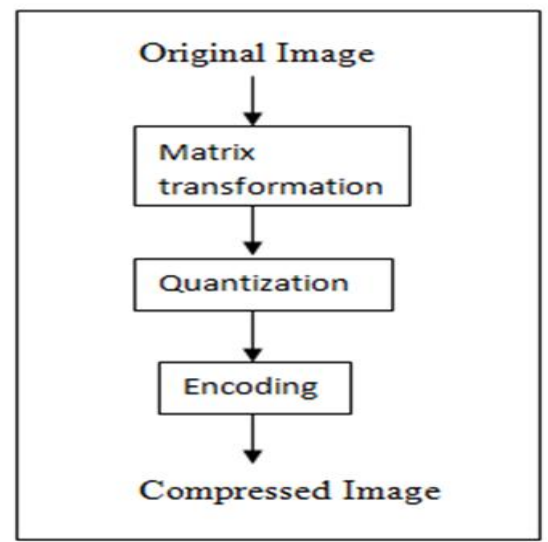

Fig-2: Lossy compression process
Matrix transformation: Matrix transformation is performed for the transformation of input data to the required approach. Algorithms like DCT is used in this step. The output of this algorithm is lossless when compared with its input.

Quantization: Quantization is the rounding - off process. Quantization is a step that has its results as lossy when compared with its input. Quantization process is irreversible. So it is not present in decompression.

Encoding: This step encodes the quantized input to be suitable to store. The output of this step is compressed when compared with its input. Encoding is the key step in any compression process. Other steps are preprocesses to encoding.

\section{COMPRESSION TECHNIQUES}

There are a large number of encoding techniques separately for lossy and lossless compressions, in which, the following are most important and used in many compression algorithms

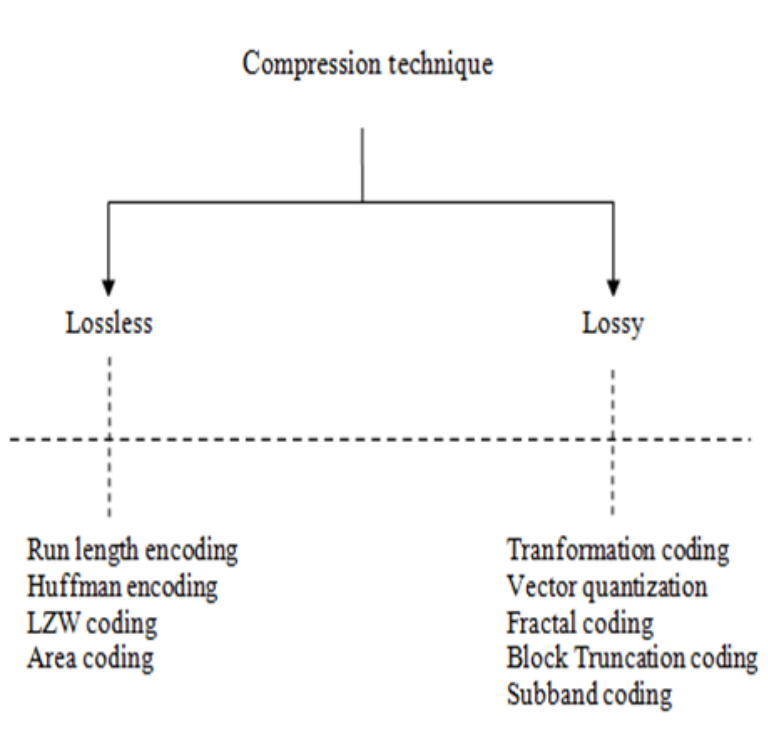

Fig-3: Lossless and Lossy compression techniques

\subsection{Lossless Compression Techniques}

\subsubsection{Run Length Encoding}

Run Length Encoding (RLE) is an entropy encoding compression technique that works on interpixel redundancy. This compression proceeds by first, finding the runs of data in the image. Runs of data refer to sequences in which same value occurs in many consecutive elements [16]. When such runs of data are identified, they are stored as a set of two values - one value being the original value that composes the run and the number of times the value is repeated. This compression algorithm is suitable for line drawings, logos and small animation files. For example consider the following example of a binary image of ' $I$ ' in black, with a white background. This is a stored in the memory as zeros and ones. 0 for white and 1 for black. 


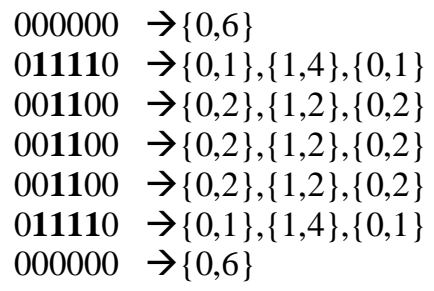

Total number of symbols in Original Image: 42

Total number of symbols in Compressed Image: 34

But this encoding scheme will not be optimal for the images without more runs.

\subsubsection{Huffman Encoding}

This is an entropy encoding technique that works on coding redundancy. This data compression algorithm encodes the data based on their probability of occurrence in the image. The elements of the image that is, the pixels are considered as symbols. All the images that contain the repetition of any particular value, does not contain it as runs. For the compression of those images, Huffman Coding is suitable rather than Run Length Encoding. For symbols that occur more frequently, smaller number of bits are assigned and others are assigned with relatively larger number of bits.

For example, in a text file we consider five symbols: A,E,I,O,U with their probability of occurrence and number of bits allotted for each of them:

Table 1: Bit allocation for various symbols

\begin{tabular}{|l|l|l|}
\hline Symbols & $\begin{array}{l}\text { Frequency of } \\
\text { occurrence }\end{array}$ & $\begin{array}{l}\text { Number of } \\
\text { bits allotted } \\
\text { to encode }\end{array}$ \\
\hline A & 12 & 6 \\
\hline E & 30 & 2 \\
\hline I & 6 & 7 \\
\hline O & 17 & 5 \\
\hline U & 22 & 3 \\
\hline
\end{tabular}

\subsubsection{LZW Coding:}

This entropy encoding technique works in the way that, various patterns in the image are noted in a dictionary and then the original image is encoded by linking the pattern in the dictionary. Once a pattern is created, whenever any pattern is encountered, the algorithm checks for whether that pattern is present already in the dictionary. If yes, then a link to that pattern in the dictionary is given. If no then that pattern is included in the dictionary and then it is linked in the original file. Hence this algorithm is known as "dictionary based compression algorithm". This encoding scheme is optimal for images having a large number of repeated patterns and not suitable for images having less number of repeated patterns since the dictionary occupies a large amount of memory space which in turn makes the file bigger.

For example Consider the occurrence of patterns "en, op, $\mathrm{ng}, \mathrm{co}, \mathrm{tr}, \mathrm{to}, \mathrm{di}$ ", in the text "entzropy encoding"
Table 2: Dictionary of patterns

\begin{tabular}{|l|l|l|l|l|l|l|}
\hline $\mathbf{1}$ & $\mathbf{2}$ & $\mathbf{3}$ & $\mathbf{4}$ & $\mathbf{5}$ & $\mathbf{6}$ & $\mathbf{7}$ \\
\hline en & $\mathbf{O p}$ & $\mathbf{N g}$ & $\mathrm{Co}$ & $\mathbf{T r}$ & to & di \\
\hline
\end{tabular}

The encoded text is: " $152 \mathrm{y} 1473$ ". The size of a pattern is taken as two. While grouping the texts, when a single element resides unable to be grouped, then that element is written as it is.

\subsubsection{Area Coding}

Area coding is an entropy encoding scheme which works on binary images. This works in the way that the whole image is divided into some blocks of same size so the resulting block may be of three types - blocks having only white pixels, blocks having only black pixels and blocks having both black and white pixels. Then the block that occurs frequently, is assigned with a 1-bit code word and the others are assigned with 2-bit code words. Thus by replacing blocks with 1-bit or 2-bit code words the file size gets reduced and thus the compression is achieved.

\subsection{Lossy Compression Techniques}

\subsubsection{Transformation Coding}

This source encoding scheme works in such a way that, the pixels in the original image is transformed into frequency domain coefficients. These frequency domain coefficients are known as transform coefficients. These coefficients have certain useful properties. For example: it has the energy compaction property due to which maximum amount of energy in the original data gets concentrated in few of the transform coefficients. These coefficients alone are selected, the remaining are deleted to achieve compression. To the selected coefficients, further processing is applied.DCT coding has been the most common approach to perform transform coding. It is also adopted in the JPEG image compression standard.

\subsubsection{Vector Quantization}

This source encoding technique is otherwise known as block quantization or pattern matching quantization[16]. The input image which contains various amplitude levels is divided into various blocks. The amplitude levels present in the input are in the form of vectors since they have both magnitude and direction. Consider an i-dimensional vector $\left[x_{1}, x_{2}, \ldots, x_{i}\right]$. It is matched to a of $\mathrm{j}$-dimensional vectors $\left[y_{1}, y_{2}, \ldots, y_{j}\right]$ with $\mathrm{j}<\mathrm{i}$. Vector space y contains the quantized values of the vector space $\mathrm{x}$. In quantization phase, the vectors are quantized in whole as a block instead of quantizing each sample. Thus this has the density matching property due to which some errors occur and these errors are inversely proportional to the density of the blocks. The quantized values of the vector space are stored as code words in a codebook. Only the index of the codeword in the codebook is used and thus compression is achieved through this also. 


\subsubsection{Fractal Coding}

This source encoding technique is useful in encoding bitmaps. Fractal coding works on the principle that real world input data contains many fractals. The similar patterns in the images getting repeated itself are known as fractals. Mapping of fractals does not require exact matches but is based on approximate matches. Thus replacing fractals occupy less memory space and due to approximate matching of fractals the compression achieved is lossy. This gives a good quality of compression even in higher compression ratios when compared with other lossy compression techniques because other techniques aim in discarding the redundant data while fractal encoding does not aim in discarding data. Fractal coding is an asymmetric technique which needs more time for compression then decompression because the process of compression needs to find the various fractals in the image using millions of iterations and the process of decompression simply needs to translate back the fractals into bitmaps. The parameters that affect the compression time and the output file size are resolution of input image, compression ratio, quality of output image. Fractal image representation is described mathematically as an Iterated Function System(IFS). Let us consider a binary image $R_{b}$ as a subset of $\mathbb{R}^{2}$. Now we want to find the IFS of the image. IFS can be described as a set of mappings $\{\mathrm{fl}, \mathrm{f} 2, \ldots, \mathrm{fN}\}$ such that $f_{i}: \mathbb{R}^{2} \rightarrow \mathbb{R}^{2}, 1 \leq i \leq n$. The IFS defines a $2 \mathrm{D}$ set $\mathrm{S}$ as a fixed point of the $\mathrm{H}$ operator. $\mathrm{H}$ operator stands for Hutchinson operator

$$
H(Z)=\bigcup_{j=1}^{N} f_{j}(Z), \quad Z \subset \mathbb{R}^{2} \quad \rightarrow(D)
$$

$\mathrm{S}$ is the unique set satisfying $\mathrm{H}(\mathrm{S})=\mathrm{S}$. This shows that $S=f_{1}(S) \cup f_{2}(S) \cup \ldots \cup f_{N}(S)$. So it is clear that IFS is the fractal representation of $\mathrm{S}$. Set $\mathrm{S}$ can be recovered using Fixed point iteration (FPI). FPI says that for any nonempty initial set $Z_{0}$, the iteration $Z_{k-1}=H\left(Z_{k}\right)$ gives the value of $\mathrm{S}$. In case of grayscale images, same steps are followed but the image $R$ is considered as the subset of $\mathbb{R}^{3}$.

\subsubsection{Block Truncation Coding:}

In this source encoding technique, image is divided into non overlapping blocks of pixels. Threshold value is determined for every block. Here the threshold value is the mean of the values in the block. It is denoted as $\bar{r}$

$$
\bar{r}=\frac{1}{N} \sum_{i=1}^{N} r_{i}
$$

$r_{i}$ is the gray value of the pixels, $\mathrm{N}$ is the total number of pixels in the block.

The values greater than $\overline{\mathrm{r}}$ are replaced by 1 , the values less than $\overline{\mathrm{r}}$ are replaced by 0 and the pixels having same value as $\overline{\mathrm{r}}$ are replaced by either 0 or 1 based on the requirement of the compression algorithm implementing this. Now we have partial output. We have the image which is separated as segments. Each segment now contains zeros and ones. Reconstruction value of these blocks is found out. Reconstruction is made using two values, $\mathrm{k}$ and $\mathrm{l}$

$$
\mathrm{k}=\overline{\mathrm{r}}-\sigma \sqrt{\frac{\mathrm{p}}{\mathrm{N}-\mathrm{p}}}
$$

And

$$
l=\bar{r}+\sigma \sqrt{\frac{N-p}{p}}
$$

Where $\mathrm{q}$ is the number of pixels having their value as one and $\sigma$ is the standard deviation, computed using the formula,

$$
\sigma=\left\{\frac{1}{m} \sum_{i=1}^{m} x_{i}-\bar{x}^{2}\right\}^{\frac{1}{2}}
$$

\subsubsection{Subband Coding}

In this source encoding technique, the input frequency band is divided into different sub-bands using digital filter bank. Consider an input $R(n)=\left(r_{1}, r_{2}, \ldots, r_{n}\right)$. Then it is separated into $\operatorname{Rx}(\mathrm{n})=\left(\mathrm{rx}_{1}, \mathrm{rx}_{2}, \ldots, \mathrm{rx}_{\mathrm{n}}\right)$ and $\mathrm{Ry}(\mathrm{n})=$ $\left(\mathrm{ry}_{1}, \mathrm{ry}_{2}, \ldots, \mathrm{ry}_{\mathrm{n}}\right)$

Where

$$
\mathrm{rx}_{\mathrm{i}}=\frac{\mathrm{r}_{\mathrm{i}}+\mathrm{r}_{\mathrm{i}-1}}{2}, \mathrm{ry}_{\mathrm{i}}=\frac{\mathrm{r}_{\mathrm{i}}-\mathrm{r}_{\mathrm{i}-1}}{2} \rightarrow(\mathrm{I})
$$

for all $2 \leq \mathrm{i} \leq \mathrm{n}$. We have $\mathrm{rx}_{1}=\mathrm{r}_{1}$ and $\mathrm{ry}_{1}=0$ $\mathrm{n}$ is the number of frequency bands

Again $r_{i}$ can be recovered using the formula

$$
r_{i}=r x_{i}+r y_{i}
$$

This digital filtration is based on the separation of low and high frequencies. Thus these filters act as the low pass and high pass filters. For further separation, each of the subbands is applied with low pass and high pass filters. This compression technique works to remove the psychovisually redundant information and hence some of the high frequency data is removed. Then each of the sub-bands is quantized separately. As the final step, the encoding of the bands is performed. The encoding technique that is suitable for each of the sub-bands is applied without being interrupted by others.

\section{CONCLUSION}

Thus from this study on digital image compression, we are able to get an idea of various types of redundancies that must be handled while compressing an image, various compression techniques followed and their approach to compress an image. Each technique follows its own way of compression and so they have specific application area. The selection of compression type plays an important role in the success of any image based application. 


\section{REFERENCES}

[1]. A study of various image compression techniques, Sonal, Dinesh Kumar, National Conference on challenges \& Opportunities in Information Technology 2007, RIMT-IIET, Punjab.

[2]. Analysis of Various Image Compression Techniques, G.M.Padmaja, P.Nirupama, ARPN journal of science and

Technology, VOL 2, NO 4, May 2012, pp.371 - 376

[3]. Fundamentals of Digital Image Processing, S.Annadurai, R.Shanmugalakshmi, Pearson Education India, October 06, 2006.

[4]. R.C.Gonzalez and R.E WOODS, Digital Image processing.Addison-Wesley Pub co., 1993

[5]. T. W. Fry, Hyper Spectral Image Compression on Reconfigurable Platforms, Master Thesis, University of Washington, Seattle, Washington, 2001.

[6]. T. Cover and J. Thomas, Elements of Information Theory. New York: John Wiley \& Sons, Inc., 1991.

[7]. Subramanya S.R, "Image Compression Technique,"Potentials IEEE, Vol. 20, Issue 1, pp 19-23, Feb-March 2001.

[8]. Affable Compression through Lossless ColumnOriented Huffman Coding Technique, Punam Bajaj, Simranjit Kaur Dhindsa, IOSR Journal of Computer Engineering (IOSR-JCE) e-ISSN: 2278-0661, p- ISSN: 2278-8727Volume 11, Issue 6 (May. - Jun. 2013), PP 89-96 [9]. TMT Quantization Table Generation Based on Psychovisual Threshold for Image Compression, Ferda Ernawan, Nur Azman Abu and Nanna Suryana, 2013 International Conference of Information and Communication Technology (ICoICT), PP 202-207

[10]. Absolute Moment Block Truncation Coding and Its Application to Color Images, Maxim D. Lema and Robert Mitchell, IEEE Transactions on Communications, vol. com32, No. 10, October 1984, PP 1148 - 1157

[11]. Lecture notes of Image Processing and Video Compression series[2005] by Prof. Amar Mukherjee, Weifeng Sun downloaded http://web.cecs.pdx.edu/ mperkows/

[12]. A vector Quantization Approach to Speaker Recognition, F. K. Soong, A. E. Rosenberg, L. R. Rabiner, B. H. Juang, AT\&T Bell Laboratories, Murray Hill, New Jersey

[13]. Image Compression and its Various Techniques, Dr. Ajit Singh, Meenakshi Gahlawat, Intenational Journal of Advanced Research in Computer Science and Software Engineering, Vol 3, Issue 6, June 2013.

[14]. Fractal Image Compression, Michael F. Barnsley, Notices of the Ams, Vol 43, No. 6, PP 657 - 662, June 1996. 